\title{
Strong coupling for fluid structure interaction problems
}

\author{
Nicolas Capron* — Gilmar Mompean *,** _ Hassan Naji *,** \\ * Laboratoire de Mécanique de Lille \\ LML UMR 8107 Université des Sciences et Technologies de Lille/CNRS \\ Avenue Paul Langevin, F-59655 Lille \\ nicolas.capron@ed.univ-lille1.fr \\ ** Ecole Universitaire Polytechnique de Lille \\ Avenue Paul Langevin, F-59655 Lille \\ \{gilmar.mompean,hassan.naji\}@polytech-lille.fr
}

\begin{abstract}
The computation of fluid forces acting on a rigid or deformable structure constitutes a major problem in fluid structure interaction. However, the majority of numerical tests consists in using two different codes to separately solve pressure of the fluid and structural displacements. In this paper, a monolithic with an ALE formulation approach is used to implicitly calculate the pressure of an incompressible fluid applied to the structure. The projection method proposed by Gresho is used to decouple the velocity and pressure.

RÉSUMÉ. Le souci majeur des problèmes d'interaction fluide-structure est le calcul des forces fluides qui agissent sur une structure rigide ou déformable. Cependant, la majorité des essais numériques consiste à utiliser deux codes différents pour résoudre séparément la pression du fluide des déplacements de la structure. Dans cet article, un code monolithique avec une approche de formulation ALE est employé pour calculer implicitement la pression d'un fluide incompressible appliquée à la structure. La méthode de projection proposée par Gresho est utilisée pour découpler la vitesse et la pression.

KEYWORDS: fluid structure interaction, projection method, ALE.

MOTS-CLÉS : interaction fluide-structure, méthode de projection, ALE.
\end{abstract}

DOI:10.3166/REMN.16.477-490@ 2007 Lavoisier, Paris. Tous droits réservés

REMN - 16/2007. Fluid structure interaction, pages 477 to 490 


\section{Introduction}

A computational procedure is developed to solve problems of viscous incompressible flows interacting with rigid or deformable structure. The arbitrary Lagrangian Eulerian method (ALE) is used to move the internal fluid nodes whereas the boundary fluid nodes move with the structure. The coupling of the mesh motion equations and the fluid equations is essentially done through contact surface boundary conditions. In continuum mechanics, two descriptions are considered for the motion in a continuum media:

- the first is the Eulerian description, where we focus attention on a particular volume in space. The volume is fixed in with respect to a laboratory frame, and we study the fluid as it passes through the fixed volume. The description is one in which the fluid is continuously renewed inside the volume, the Eulerian description is not the simplest in which the basic equations of fluid motion can be formulated. A convective term is introduced to express the material time derivative in the reference configuration. The convective term gives a nonsymmetrical form of the Galerkin formulation. Since the computational domain is fixed, the Eulerian description has the advantage of preserving the mesh regularity;

- the second is the Lagrangian description, in which we identify and follow a particular region of fluid. The volume of fluid changes in shape, while the total mass remains constant. In the Lagrangian description, the mesh of the computational domain moves with the particle fluid velocity. In the Lagrangian description, the motion of the mesh may lead to element entanglement; this description is preferred for problems with small motion.

In this paper, we present the algorithm of a monolithic code which permits to compute the structural displacement, fluid velocity and pressure at the same time. In order to solve the problem, we use a finite element formulation to solve the governing equations for the structure and the Navier-Stokes equations with a Lagrangian formulation. If we solve the governing equations for the fluid in Eulerian or Arbitrary Langrangian Eulerian (ALE) formulation, we use the "split" operator described in Section 4. In order to solve fluid structure interaction problems, we have to compute the pressure acting on the structure. The projection method, introduced initially by Chorin and Temam (1968) and proposed by Gresho (1990) is implemented to meet this requirement. The numerical example studied in this paper shows the interest of an implicit pressure for this type of problems. This paper is organized as follows: in the Section 2, the ALE formulation is described. In the Section 3, the governing equations are presented. The Sections 4 and 5 are devoted respectively to the description of the structural and fluid analysis algorithm. In the Section 6, the numerical implementation is described and a numerical test is proposed in the Section 7. More precisely, we validate the algorithm on the case of the flow between two cylinders, numerical results that indicate the effectiveness of the approach are presented. 


\section{The ALE description}

The ALE description for incompressible viscous flows has been developed by Hughes et al., (1981), to solve free surface flows and fluid structure interaction problems. A general kinematics theory was developed by Hughes, which serves as the basis of the Lagrangian-Eulerian description. For this purpose, the authors define three domains in space, and mappings from one domain to the other. The first one, called the spatial domain, is considered as the domain on which the fluid problem is posed. The spatial domain is generally in motion, because of moving boundaries. The second domain, called the material domain, is to be thought of as the domain occupied at time $t=0$ by the material particles which occupy the spatial domain at time $t$. The third domain, called the reference domain, is defined as a fixed domain throughout. From these domain descriptions, we can see that the Eulerian description is obtained when the spatial domain coincides with the reference domain, whereas the Lagrangian reference is obtained when the material domain coincides with the reference domain.

Both the material and spatial domains are generally in motion with respect to the reference domain; it is convenient to express the material time derivative of a physical property $\phi$ in the reference configuration.

$$
\dot{\phi}=\phi_{, t}+c . \nabla \phi
$$

where $\phi$ is the material time derivative, and $\phi_{t}$ is the time derivative when freezing coordinates in the reference domain, $c$ is the convective velocity.

$$
c=v-v^{m}
$$

$v$ is the fluid velocity, and $v^{\mathrm{m}}$ is the mesh velocity. In the Eulerian description, the mesh velocity is zero, $v^{\mathrm{m}}=0$, whereas in the Lagrangian description $v^{\mathrm{m}}=v$ and $c=0$.

In the ALE formulation, the mesh nodes move with an arbitrary velocity. The choice of the mesh velocity constitutes one of the major problems with the ALE description. Different techniques have been developed for updating the mesh in a fluid motion, depending on the fluid domain. For problems defined in simple domains, the mesh velocity can be deduced through a uniform or non uniform distribution of the nodes along straight lines ending at the moving boundaries. For general computational domains, the mesh velocity is computed through partial differential equations, with appropriate boundary conditions (see Longatte et al., 2003). A more general diffusion equation can be used for the mesh velocity:

$$
\operatorname{div}((1+\tau) \nabla v)=0
$$


where $\tau$ is a non dimensional function given on each element by:

$$
\tau=\frac{\Delta_{\max }-\Delta_{\min }}{\Delta_{\text {elem }}}
$$

in which $\Delta_{\text {elem }}$ represents the area of the current element. The parameter $\tau$ is designed in order to prevent distortion of small elements.

\section{Governing equations}

The Lagrangian formulations are frequently used to solve the structural problems. Indeed, displacements of the nodes and the elements on a Lagrangian mesh correspond to the movements of material. The material edges always coincide with the edges of the elements. Thus, if the material sharply becomes deformed, the mesh is subjected to large distortions. In general, the structural deformations are weak so that the Lagrangian mesh remains regular and is not subjected to distortions. The boundary conditions are easily imposed because the edges of the mesh represent the limits of the physical domain during calculation. For these reasons, the Lagrangian formulations are much appreciated. In the Cartesian coordinate system, the displacement of the structure $u$ in a domain $\Omega_{S}$ (see Figure 1) is governed by

$$
\rho_{S} \frac{\partial^{2} u_{i}}{\partial t^{2}}=\sigma_{i j}(u)_{, j}+\rho_{S} g_{i}
$$

with initial and boundary conditions

$$
u_{i}=\hat{u}_{i} \quad \text { on } \delta \Omega_{D S} \times[0, T]
$$

in which, $\sigma_{\mathrm{ij}}, \rho_{S}, \mathrm{~g}_{\mathrm{i}}$ and $\hat{u}_{i}$ stand for, respectively, the Cauchy stress tensor, the structure density, gravity acceleration components and imposed displacement components. $\delta \Omega_{D S}$ is the structural displacement boundary region. $T$ is the final time of the run.

Two points of view are generally considered to describe the movement of a fluid. The first is Lagrangian where the motion of the mesh follows that of the fluid. The disadvantage of this description is to generate large mesh distortions. The second is Eulerian and consists in studying the movement of the fluid in fixed positions. The domain of study is fixed and the fluid is updated constantly in this one. This method introduces a term of convection into the equations to be solved. It avoids the great distortions of mesh. However, the difficulty is deferred to the interface where it is difficult to represent the boundary conditions for a problem of interaction fluid structure. 


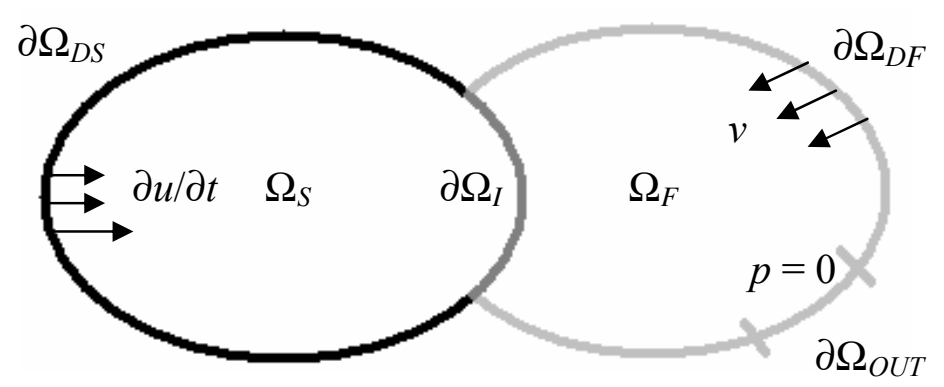

Figure 1. Fluid and structure domains

So, we made recourse to a mixed formulation. This later is the ALE method which combines at the same time Eulerian and Lagrangian descriptions to describe the movement of the fluid particles. In this framework, the velocity of the incompressible viscous fluid in a domain $\Omega_{F}$ is characterized by the mass and momentum conservation laws such that

$$
\begin{aligned}
& v_{i, i}=0 \quad \text { in } \Omega_{F} \times[0, T] \\
& \frac{\partial v_{i}}{\partial t}+\left(v_{j}-v_{j}^{m}\right) v_{i, j}-\frac{1}{\rho_{F}} \tau_{i j, j}=g_{i} \quad \text { in } \Omega_{F} \times[0, T]
\end{aligned}
$$

where $v_{i}$ and $\rho_{F}$ indicate, respectively, the flow velocity components and the fluid density. The term $v^{m}$ represents the velocity of the mesh. If $v^{m}=0$, we obtain the Eulerian formulation because the convective velocity of the mesh is nil. If $v^{\mathrm{m}}=v$, we obtain the Lagrangian formulation for which the convective velocity is the fluid velocity. The quantity $v_{A L E}=v-v^{m}$ is the relative velocity and the stress tensor $\tau$ is commonly defined by:

$$
\tau_{i j}=\mu_{F}\left(v_{i, j}+v_{j, i}\right)-p \delta_{i j}
$$

where $\mu_{F}$ is the dynamic viscosity.

The momentum equation is to be solved with the initial condition and the boundary conditions on the boundary region $\delta \Omega_{D F}$ :

$$
\begin{aligned}
& v_{i}(0)=0 \quad \text { in } \Omega_{F} \\
& v_{i}=\hat{v}_{i} \text { on } \delta \Omega_{D F} \times[0, T]
\end{aligned}
$$

where $\hat{v}_{i}$ are the imposed velocity components on $\delta \Omega_{D F}$. 
The boundary conditions on the fluid structure interface $\delta \Omega_{I}$ are given by:

$$
v_{i}=\frac{\partial u_{i}}{\partial t} \text { on } \delta \Omega_{I} \times[0, T]
$$

and $p=0$, on the outflow boundary $\delta \Omega_{O U T}$.

\section{Structural analysis algorithm}

To model the structural behaviour, we use the elastic constitutive law. So the Cauchy stress tensor is integrated incrementally in time (see Hallquist, 1998):

$$
\sigma_{i j}^{n+1}=\sigma_{i j}^{n}+\dot{\sigma}_{i j} \Delta t
$$

where the superscript $n$ indicates the value of the quantity at time $t=n \Delta t$ and the dot denotes the material time derivative given by:

$$
\dot{\sigma}_{i j}=\sigma_{i j}^{\nabla}+\sigma_{i k} \omega_{k j}+\sigma_{j k} \omega_{k i}
$$

in which $\omega$ is the spin tensor

$$
\omega_{i j}=\frac{1}{2}\left(\frac{\partial u_{i}}{\partial x_{j}}-\frac{\partial u_{j}}{\partial x_{i}}\right)
$$

and $\sigma^{\nabla}$ is the Jaumann stress tensor

$$
\sigma^{\nabla}=2 \mu \dot{\varepsilon}+\lambda \operatorname{Tr}(\dot{\varepsilon}) I d
$$

where $\mu, \lambda$ are the Lamé coefficients and $\operatorname{Tr}$ is the trace. The strain tensor $\varepsilon$ is defined as follows:

$$
\varepsilon_{i j}=\frac{1}{2}\left(u_{i, j}+u_{j, i}\right)
$$

Introducing the Young modulus $E$ and the Poisson number $v$ which are defined as: 


$$
\begin{aligned}
& E=\frac{\mu(2 \mu+3 \lambda)}{\mu+\lambda} \\
& v=\frac{\lambda}{2(\mu+\lambda)},
\end{aligned}
$$

the Jaumann stress tensor can be written:

$$
\sigma^{\nabla}=\frac{E}{1+v}\left(\dot{\varepsilon}+\frac{v}{1-2 v} \operatorname{Tr}(\dot{\varepsilon}) I d\right)
$$

So the computation of the displacement $u$ is split into three steps:

- Strain tensor. The first consists in calculating the strain tensor $\varepsilon$ with the displacement obtained at the last time step.

$$
\dot{\varepsilon}_{i j}^{n+1}=\frac{1}{2}\left(\dot{u}_{i, j}^{n}+\dot{u}_{j, i}^{n}\right)
$$

- Cauchy stress tensor. With the new value $\dot{\varepsilon}_{i j}^{n+1}$ and by neglecting rotations, the Cauchy stress tensor $\sigma$ is computed

$$
\sigma_{i j}^{n+1}=\sigma_{i j}^{n}+\frac{E}{1+v}\left(\dot{\varepsilon}_{i j}^{n+1}+\frac{v}{1-2 v} \operatorname{Tr}\left(\dot{\varepsilon}^{n+1}\right) \delta_{i j}\right) \Delta t
$$

- Displacement update. The double integration of the residue gives:

$$
\begin{aligned}
& \dot{u}_{i}^{n+1}=\dot{u}_{i}^{n}+\frac{\Delta t}{\rho_{S}}\left(\sigma_{i j, j}^{n+1}+g_{i}^{n}\right) \\
& u_{i}^{n+1}=\dot{u}_{i}^{n+1} \Delta t
\end{aligned}
$$

where the dot is the time derivative.

Numerical computational of Equations [21] to [24] is performed with a finite element method. 


\section{Fluid analysis algorithm}

It is well known that the main difficulties arising in the numerical solution of the convection-diffusion equations are due to their no-self-adjoint character. The standard Galerkin method leads to no physical spatial oscillations when applied to the high convective case. To preclude such anomalies, the most popular method being the use of upwind differencing on the convective term via Petrov-Galerkin methods (see, for example, Heinrich et al., Heinrich and Zienkiewicz, Belytscho et al.). Although theses methods are precise and stable, we will use a "split" method which is a simple mean to obtain a robust and effective formulation. This time-split method decomposes the time step into two phases:

- phase 1 is a solution of the Lagrangian equations of motion (advection terms are nil) updating the velocity field by the effects of all forces. For the fluid, the velocity-pressure formulation of the discretized problem is decoupled by the projection method (for more details, see Medic and Mohammadi (1999) and Cho and Lee (2003));

- phase 2 adds advection contributions, and is required for runs that are Eulerian or contain some relative motion of mesh and fluid.

In order to effectively solve the pressure and velocities satisfying the continuity constraint [7] for the phase 1, we adopt the fractional method proposed by Gresho (1990). The idea of these methods is to decouple the velocity $v$ and the pressure $p$. These are based on a resolution in three steps of the Navier-Stokes equations.

Hereafter, we describe briefly the above method in Langrangian formulation.

- Intermediate velocity. The first step consists in calculating an intermediate velocity $v^{*}$, solution of the Naviers-Stokes equation without taking into account the continuity constraint.

$$
\begin{aligned}
& v_{i}^{* n+1}=v_{i}^{n}+\Delta t\left(\frac{\mu_{F}}{\rho_{F}} v_{i, j j}^{n}-\frac{1}{\rho_{F}} p_{, i}^{n}+g_{i}^{n}\right) \text { in } \Omega_{F} \\
& v_{i}^{* n+1}=\frac{\partial u_{i}^{n}}{\partial t} \text { on } \partial \Omega_{I}
\end{aligned}
$$

- Projection. As the velocity $v^{*}$ does not yet satisfy the incompressibility condition [7], it is projected on a divergence free space to get an adequate approximation of the velocity. This is obtained from:

$$
v_{i}^{*}=v_{i}+\frac{\Delta t}{\rho_{F}} \Delta p_{, i}
$$

with $v_{i, i}=0$ in $\Omega_{F}$. The term $\Delta p$ is a corrective pressure. 
The second step consists in deriving a Poisson equation for the pressure $\mathrm{p}$. In fact, by taking the divergence of the Equation [27] and using the incompressibility condition [7], we obtain:

$$
\frac{1}{\rho_{F}} \Delta p_{, i i}^{n+1}=\frac{1}{\Delta t} v_{i, i}^{* n+1} \text { in } \Omega_{F}
$$

Once the corrective pressure $\Delta p^{\mathrm{n}+1}$ has been determined, the final velocity field is obtained from the intermediate velocity $v^{*}$ and $\Delta p$ :

$$
v_{i}^{n+1}=v_{i}^{* n+1}-\frac{\Delta t}{\rho_{F}} \Delta p_{, i}^{n+1} \quad \text { in } \Omega_{F}
$$

- Pressure update. Since $v$ is the physical velocity, the pressure $p$ can be given from $\Delta p$.

$$
p^{n+1}=p^{n}+2 \Delta p^{n+1}
$$

For the phase 2, we used a first order Godunov method: the Donor Cell (see Benson, 1992 and Amsden et al., 1980). This step is bypassed for a purely Lagrangian calculation. In all other cases (Eulerian and ALE calculation) the relative velocity $v_{A L E}$ is not nil, and we must calculate the flux of momentum between cells. For each cell (see Figure 2), we calculate the volume swept out by each of faces relative to their Lagrangian positions $x_{L}$. According to the sign of these volumes, we add or remove momentum to the cell.

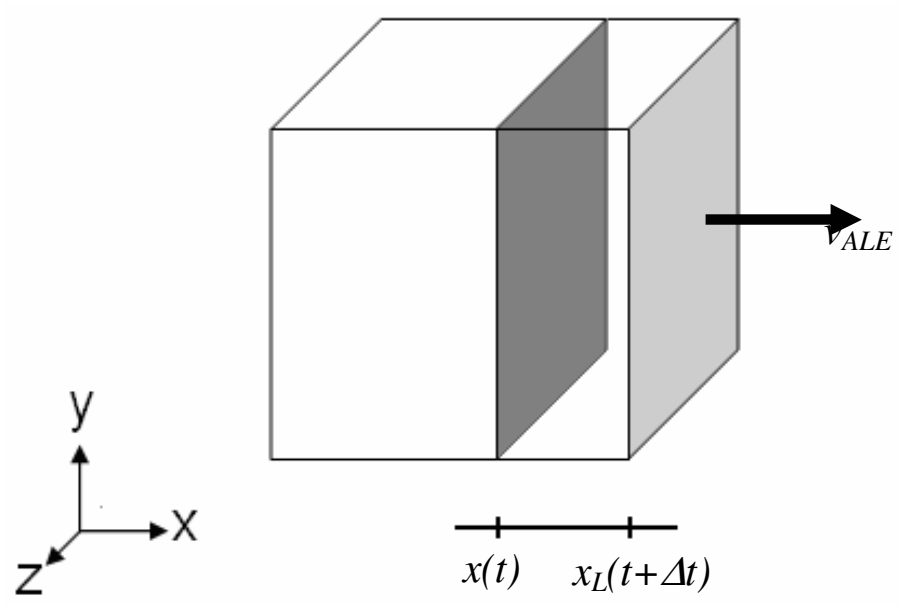

Figure 2. Volume swept out by a face in one direction 


\section{Numerical implementation}

In the course of the Langrangian phase, we compute structural displacements and intermediate velocities necessary to the projection method. To obtain displacements and velocities, we compute nodal forces from respectively the Equations [25] and [26]. This allows us to use the same method to solve the structural behaviour and the liquid dynamic response. The difference between the structural algorithm and the fluid algorithm is the computation of stress tensors $\sigma$ of the structure and $\tau$ of the fluid with the laws (see Equations [20] and [9]) which are different. Then, we solve the pressure form the Equations [28] and [30]. So, we obtain the velocity which is solenoidal $(\operatorname{div}(v)=0)$. For the Lagrangian nodes, we move the domain to update the coordinates of the nodes. And for the other nodes, we compute the momentum flux of the cell in order to update the velocity.

The numerical algorithm is sketched on figure 3.

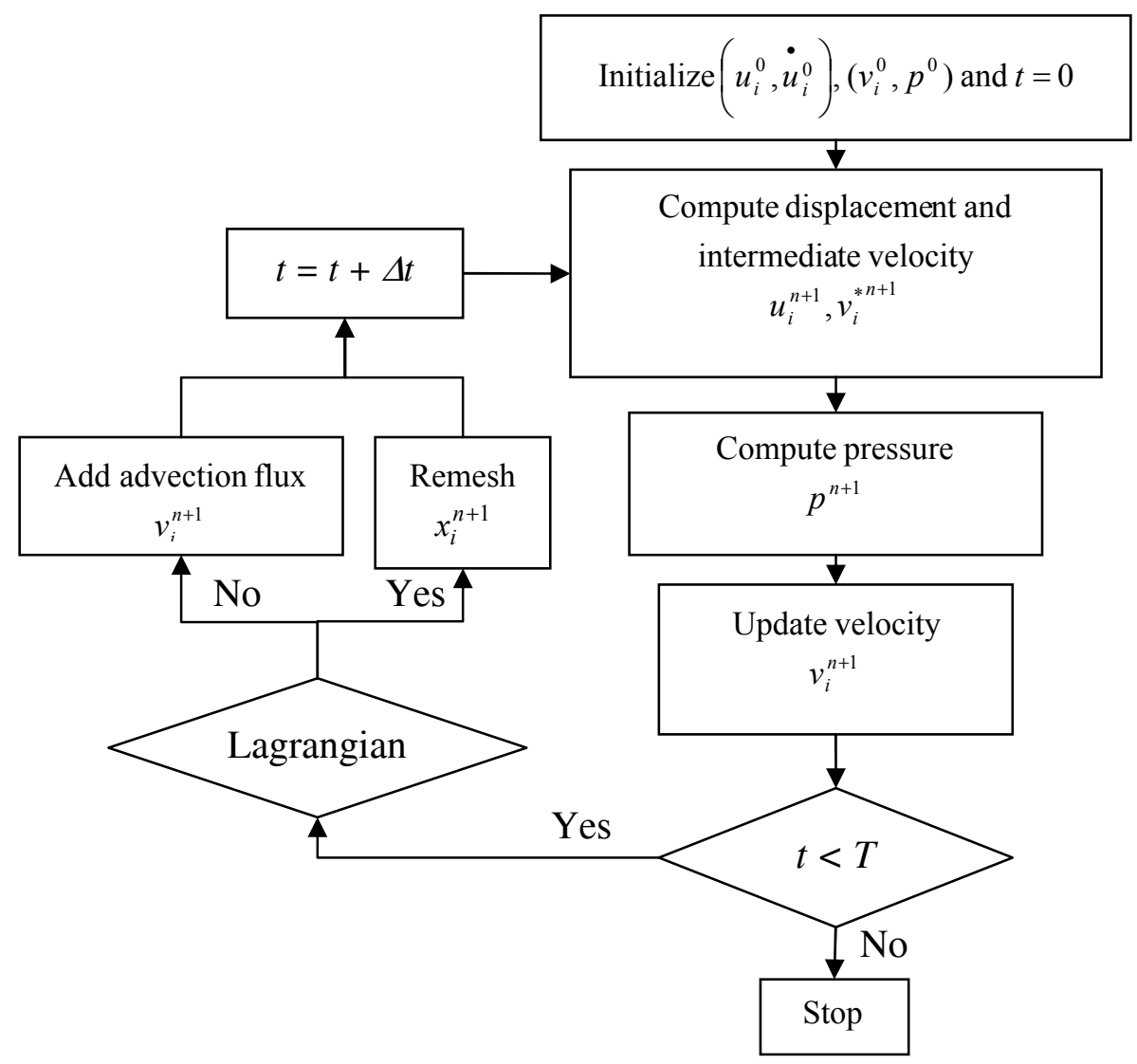

Figure 3. Flowchart for the time-incremental fluid structure numerical analysis 


\section{Numerical results}

To illustrate this numerical method, we study the case of a confined flow between two cylinders which are considered as infinite (see Figure 4). The diameter of the outer cylinder is $D=5,5 \cdot 10^{-2} \mathrm{~m}$ and that of the inner cylinder is $d=2,2 \cdot 10^{-2} \mathrm{~m}$. The thickness of the walls is $e=10^{-3} \mathrm{~m}$. The structural density, the Young modulus and the Poisson number are respectively $\rho_{S}=2700 \mathrm{~kg} \cdot \mathrm{m}^{-3}, E=69000 \mathrm{MPa}$ and $v=0,3$. The fluid density and the kinematical viscosity are respectively $\rho_{F}=1000 \mathrm{~kg} \cdot \mathrm{m}^{-3}$ and $\mu=1,7545 \cdot 10^{-2} \mathrm{~m}^{2} \cdot \mathrm{s}^{-1}$. Only the inner cylinder is excited, the other is fixed. Its velocity has the form:

$$
v(x, y, z, t)=v_{x}(t)=A \sin (\pi f t)
$$

with an amplitude $A=10^{-3} \mathrm{~m}$ and a frequency $f=38,7 \mathrm{~Hz}$.

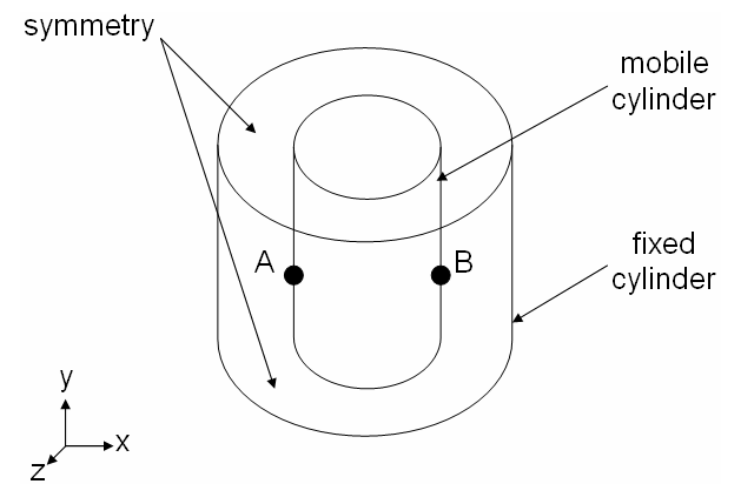

Figure 4. Problem description

In this example, $\delta \Omega_{I}=\delta \Omega_{D S}$ and $\delta \Omega_{D F}=\delta \Omega_{O U T}=\varnothing$.

We compare our results obtained by the method described in this paragraph 6 with those obtained by ASTER-SATURN and provided by Electricité De France (EDF) (Bendjeddou, 2005). In this paper, we examine the evolution of the pressure at the points $\mathrm{A}$ and $\mathrm{B}$ which are diametrically opposite (see Figures 4 and 5). To not deform the fluid mesh and create great distortions with the structural displacement, we use an ALE mesh for the elements near the inner mobile cylinder and an Eulerian mesh for the others elements (see Figure 5). This enables us to obtain a good approximation of the pressure of the element since in this case the mesh is not crushed. 


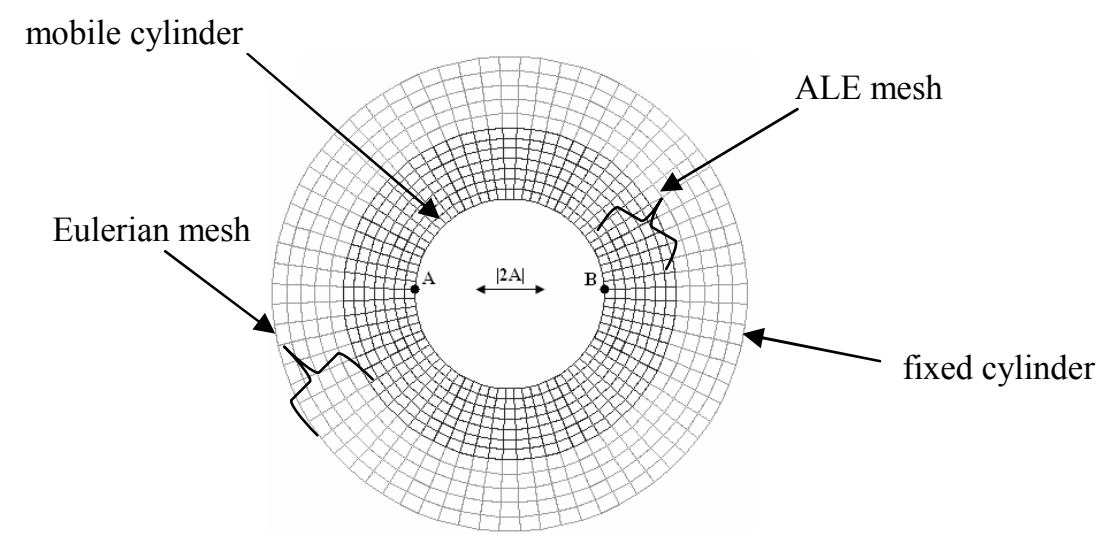

Figure 5. Computational mesh

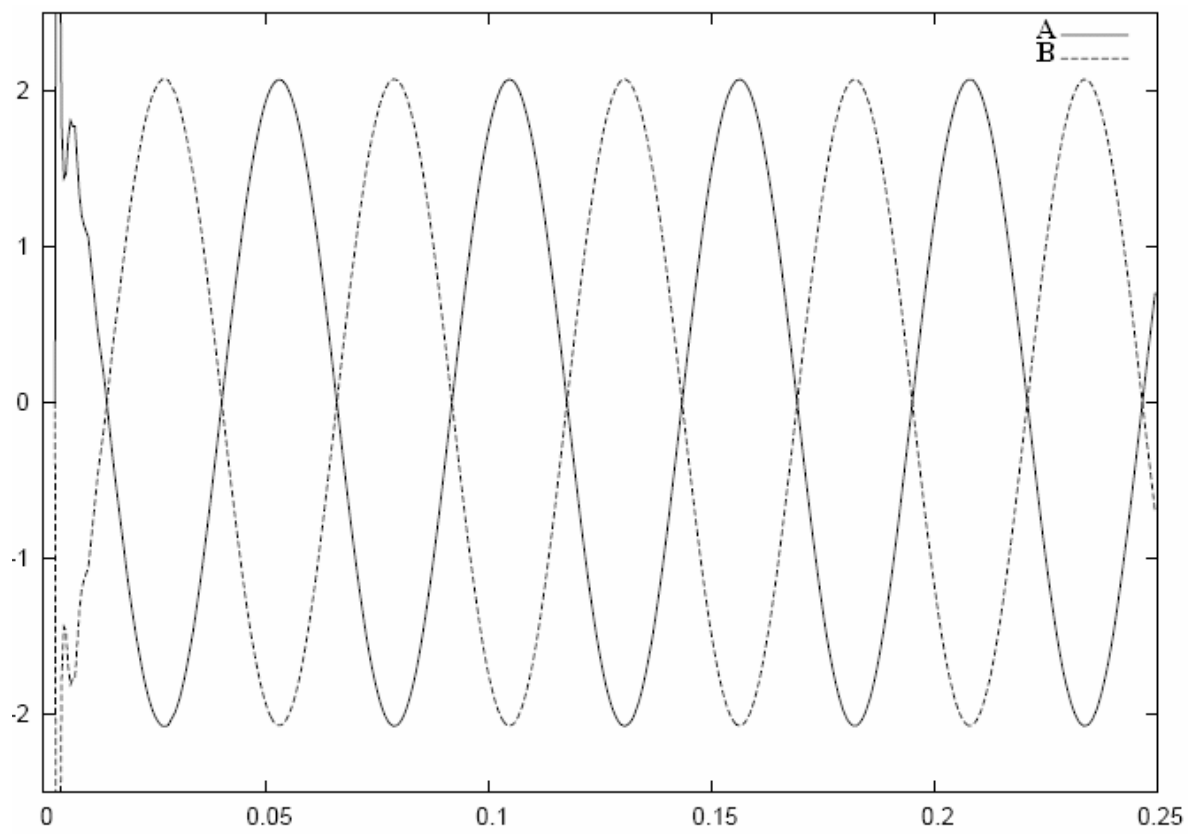

Figure 6. Pressure evolution at the points $A$ and $B$ 


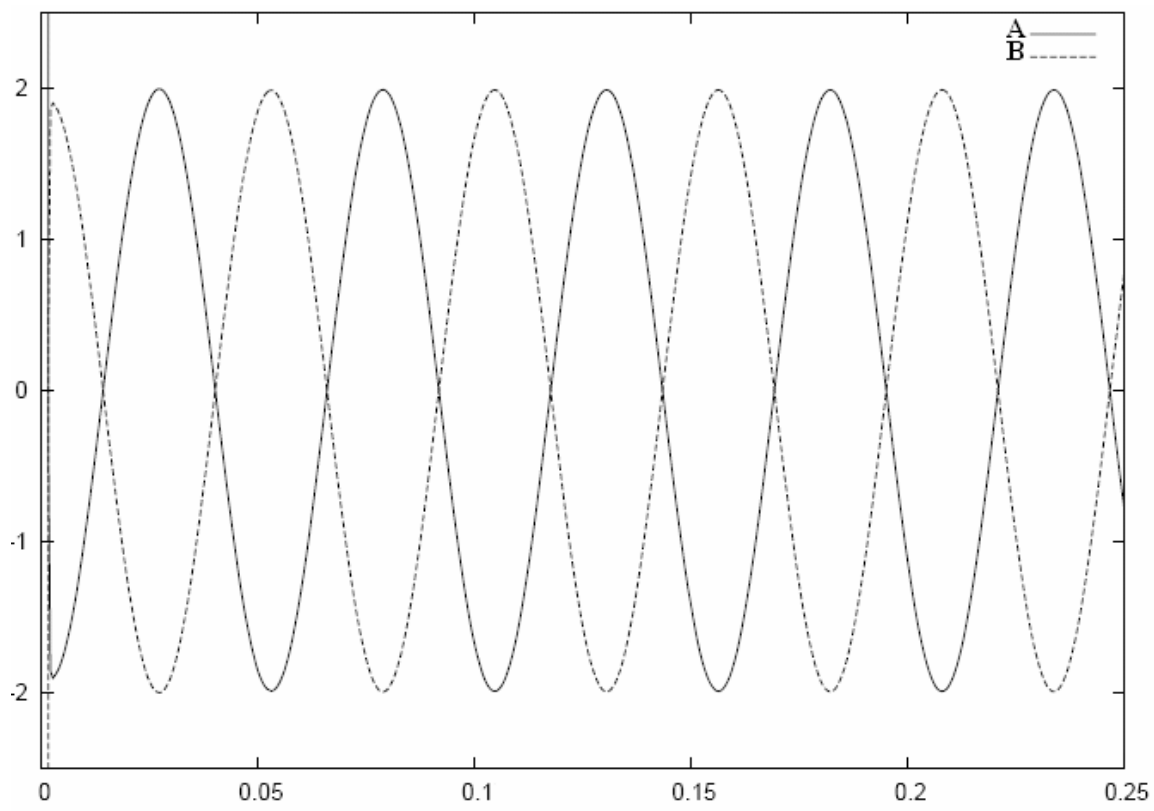

Figure 7. Pressure evolution at the points $A$ and B (ASTER-SATURNE)

Figures 6 and 7 show the pressure evolution at two points obtained by the method adopted here and ASTER-SATURNE code respectively. We can observe that our results agree well with those reported by EDF. Moreover, the frequency of the response is the same frequency as that the imposed velocity defined by the Equation [31]. This study seems to enable us to validate the present numerical method of resolution for the equations described in the paragraph 1 for the rigid body.

\section{Conclusion}

In this paper, an ALE formulation for viscous incompressible flow, and a strong coupling algorithm for fluid structure interaction problems, where structural nodes and fluid nodes are merged have been presented. A spatial finite element discretization is used to solve both fluid and structure problems. However, for the advection term of the Navier-Stokes equation, a first order Godunov method or a second Van Leer algorithm can be used. For the computation of the fluid dynamic response, the projection method defined by Gresho is implemented in order to handle the pressure term in the Navier Stokes equations. Numerical test shows that the projection method is an appropriate one for predicting fluid structure interaction 
problem. The extension of this work will be the computation of fluid forces that act on a deformable structure in order to take into account the real structural behaviour.

\section{Acknowledgements}

The authors thank F. Huvelin for providing results obtained by ASTER SATURN at EDF R\&D Chatou, France.

\section{References}

Amsden A. A., Ruppel H. M., Hirt C. W., SALE - a simplified ALE computer program for fluid flow at all speeds, Report, Los Alamos National Laboratory, 1980.

Belytscho T., Liu W. K., Moran B., Nonlinear finite elements for continua and structures, Wiley, Chichester, 2000.

Bendjeddou Z., Méthodologie pour la simulation numérique des vibrations induites par écoulements dans les faisceaux de tubes, Thèse de doctorat, Université de Lille 1, 2005.

Benson D.J., "Lagrangian and Eulerian hydrocodes", Comput. Methods Appl. Mech. Eng, 99, 1992, p. 235-394.

Cho J.R., Lee S.Y., "Dynamic analysis of baffled fule-storage tanks using the ALE finite element method", Int. Journ. Num. Meth. Fluids, 41, 2003, p. 185-208.

Chorin A.J., "Numerical solution of the Navier-Stokes equations", Math. Comp., 22, 1968, p. 745-768.

Gresho P.M., "On the theory of semi-implicit projection methods for viscous incompressible flow and its implementation via a finite element method that also introduces a nearly consistent mass matrix. Part 1: theory", Int. Journ. Num. Meth. Fluids, 11, 1990, p. 587-620.

Hallquist J.O., Theorical manual for LS-DYNA, Livermore Software Technology Corporation, Livermore, 1998.

Heinrich J.C., Huyakorn P.S., Zienkiewicz O.C., Mitchel A.R., “An upwind finite element for two-dimensional convective transport equations", Int. Journ. Num. Meth. Eng., 11, 1977, p. 131-143.

Heinrich J.C., Zienkiewicz O.C., "Quadratic finite element schemes for two-dimensional convective transport problems", Int. Journ. Num. Meth. Eng., 11, 1977, p. 1831-1844.

Hughes T.J.R., Liu W.K., Zimmerman T.K., "Lagrangian Eulerian finite element formulation for viscous flows", Comp. Meth. in Applied Mech. and Eng., 29, 1981, p. 329-349.

Longatte E., Bendjeddou Z., Souli M., “Application of arbitrary Lagrange Euler formulations to flow-induced vibration problems", ASME J. Pressure Vessel Technol., 125, 2003, p. 411-417.

Medic G., Mohammadi B., NSIKE - an incompressible Navier-Stokes solver for unstructured meshes, Research report No. 3644, march 1999, INRIA.

Temam R., Navier-Stokes equations, North-Holland, Amsterdam, 1984. 